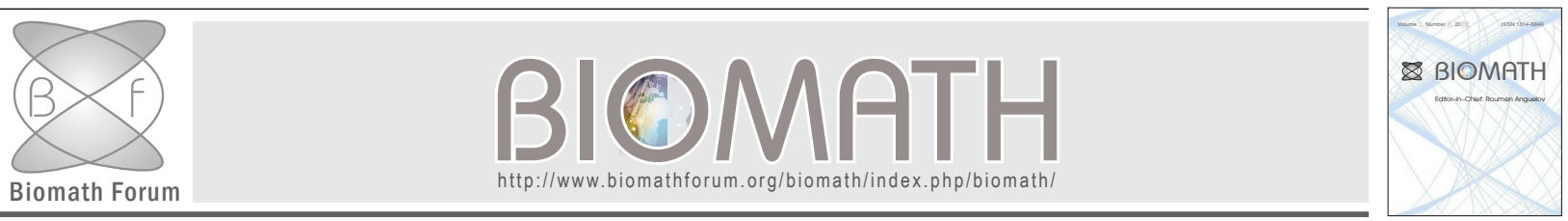

\title{
The Linear Space of Hausdorff Continuous Interval Functions
}

\author{
Dedicated to Prof. Svetoslav Markov on the occasion of his 70th birthday. \\ Jan Harm van der Walt \\ Department of Mathematics and Applied Mathematics \\ University of Pretoria \\ Pretoria, South Africa \\ janharm.vanderwalt@up.ac.za
}

Received: 11 October 2013, accepted: 26 November 2013, published: 23 December 2013

\begin{abstract}
In this paper we discuss the algebraic structure of the space $\mathbb{H}(X)$ of finite Hausdorff continuous interval functions defined on an arbitrary topological space $X$. In particular, we show that $\mathbb{H}(X)$ is a linear space over $\mathbb{R}$ containing $\mathscr{C}(X)$, the space of continuous real functions on $X$, as a linear subspace. In addition, we prove that the order on $\mathbb{H}(X)$ is compatible with the linear structure introduced here so that $\mathbb{H}(X)$ is an Archimedean vector lattice.
\end{abstract}

Keywords-Interval functions; vector space; vector lattice;

\section{INTRODUCTION}

Interval functions, and interval analysis in general, is typically associated with numerical analysis and validated computing, see for instance [22], [23]. Since the inception of the field in the 1960s, interval functions have been used extensively in mathematics and its applications, including in approximation theory [26], nonsmooth and nonlinear analysis [8], [10], [13], differential inclusions [9], [12], [16], [17], convex analysis [13], [25], nonlinear partial differential equations [5], functional analysis [6] and optimization and optimal control theory [13], [17]. Furthermore, Interval Analysis, and Set-Valued Analysis in general, are essential tools in the design and analysis of mathematical models in the life sciences. As pointed out in [21], biological dynamic systems typically involve uncertain data and / or parameters, numerical and / or inherent sensitivity and structural uncertainties which necessitate model val- idation. Problems related to these issues of uncertainty and sensitivity, including computing enclosures for sets of solutions [24] and estimation of parameter ranges [15], essentially belong to Set-Valued Analysis in general and are often addressed within the setting of Interval Analysis. We refer the reader to [21] for more details.

In [4] it was shown that the set $\mathbb{H}(X)$ of Hausdorff continuous (H-continuous) interval functions defined on an open subset $X$ of $\mathbb{R}^{n}$ is a linear space over $\mathbb{R}$ containing $\mathscr{C}(X)$ as a linear subspace. The aim of this paper is to extend this result to the most general case. In particular, we will show that $\mathbb{H}(X)$ is a linear space over $\mathbb{R}$ for every topological space $X$. As will be seen, this extension of the result in [4] requires an entirely new approach, since the methods used in [4] do not apply in the general setting considered here.

While the result as such is not entirely unexpected, the method used in the proof is of intrinsic interest. Indeed, our method reveals the essential mathematical mechanism that allows the extension of the linear operations on $\mathscr{C}(X)$ to the larger set $\mathbb{H}(X)$, namely, a minimality condition satisfied by $\mathrm{H}$-continuous functions which is (almost) preserved by pointwise interval arithmetic [3]. Furthermore, our method gives an indication of how to define algebraic operations on certain spaces of setvalued maps that take values in a general metrisable topological vector space. This problem is addressed in [7] in the case when the domain is a Baire space, while the method developed here for $\mathrm{H}$-continuous functions 
gives some direction as to how one may proceed in the case of functions defined on a general topological space.

\section{Preliminaries}

\section{A. H-continuous Functions}

For the convenience of the reader unfamiliar with Interval Analysis, we now recall those definitions and results that will be used in subsequent sections. We denote by $\mathbb{I} \mathbb{R}$ the set of real intervals, that is

$$
\mathbb{I R}=\{a=[\underline{a}, \bar{a}]: \underline{a}, \bar{a} \in \mathbb{R}, \underline{a} \leq \bar{a}\} .
$$

For a topological space $X$, we call a function $f: X \rightarrow$ $\mathbb{I} \mathbb{R}$ locally bounded if for every $x \in X$ there exists a neighbourhood $V$ of $x$ and a constant $M \in \mathbb{R}$ so that $f(y) \subseteq[-M, M]$ for every $y \in V$. The set of locally bounded functions $f: X \rightarrow \mathbb{I R}$ is denoted as $\mathbb{A}(X)$, while $\mathscr{A}(X)$ is the set of real valued locally bounded functions.

Every $f \in \mathbb{A}(X)$ may be identified with a pair of (real valued) functions $f, \bar{f} \in \mathscr{A}(X)$ by setting $f(x)=\inf f(x)$ and $\bar{f}(x)=\sup f(\bar{x}), x \in X$. Clearly $f(x)=[f(x), \bar{f}(x)]$ for each $x \in X$. Conversely, by identifying every $a \in \mathbb{R}$ with the degenerate interval $[a, a]$, we may consider $\mathscr{A}(X)$ as a subset of $\mathbb{A}(X)$.

The relation

$$
f \leq g \Leftrightarrow\left(\begin{array}{ll}
\forall & x \in X: \\
& \underline{f}(x) \leq \underline{g}(x), \bar{f}(x) \leq \bar{g}(x)
\end{array}\right)
$$

with $f=[f, \bar{f}], g=[g, \bar{g}] \in \mathbb{A}(X)$, defines a partial order on the set $\overline{\mathbb{A}}(X)$. Note that the order (1) extends the usual pointwise order on $\mathscr{A}(X)$.

The Upper and Lower Baire Operators [1], [11], [26] are mappings $S, I: \mathbb{A}(X) \rightarrow \mathscr{A}(X)$ defined by

$$
S(f)(x)=\inf \left\{\sup \{z \in f(y): y \in V\}: V \in \mathscr{V}_{x}\right\}
$$

and

$$
I(f)(x)=\sup \left\{\inf \{z \in f(y): y \in V\}: V \in \mathscr{V}_{x}\right\},
$$

respectively, with $\mathscr{V}_{x}$ denoting the set of open neighbourhoods at $x \in X$. The Graph Completion Operator $F: \mathbb{A}(X) \rightarrow \mathbb{A}(X)$ is defined by setting

$$
F(f)=[I(f), S(f)], f \in \mathbb{A}(X) .
$$

The operators $I, S$ and $F$ are monotone with respect to the order $(1)$ on $\mathbb{A}(X)$ and the pointwise order on $\mathscr{A}(X)$. That is,

$$
f \leq g \Rightarrow I(f) \leq I(g), S(f) \leq S(g), F(f) \leq F(g) .
$$

Consequently, $F$ is inclusion isotone so that

$$
f(x) \subseteq g(x), x \in X \Rightarrow F(f)(x) \subseteq F(g)(x), x \in X .
$$

Furthermore,

$$
I(I(f))=I(f), S(S(f))=S(f), F(F(f))=F(f)
$$

and

$$
I(f) \leq F(f) \leq S(f)
$$

for every $f \in \mathbb{A}(X)$, where we consider the real valued functions $I(f)$ and $S(f)$ as interval functions, as explained previously.

Using the Graph Completion Operator, the following notions of continuity of interval functions are defined, see [1], [26].

Definition 2.1: A function $f \in \mathbb{A}(X)$ is Sendov continuous (S-continuous) if $F(f)=f$.

Definition 2.2: A function $f \in \mathbb{A}(X)$ is Hausdorff conitnuous (H-continuous) if $f$ is S-continuous and $f=g$ for every S-continuous function $g$ so that $g(x) \subseteq$ $f(x), x \in X$.

The set of all S-continuous functions is denoted by $\mathbb{F}(X)$ while $\mathbb{H}(X)$ denotes the set of $\mathrm{H}$-continuous functions.

We may note that the Baire operators characterise lower and upper semi-continuity of real functions. In particular, for every $f \in \mathscr{A}(X)$ we have

$$
f \text { is lower semi - continuous } \Leftrightarrow I(f)=f
$$

and

$$
f \text { is upper semi - continuous } \Leftrightarrow S(f)=f \text {. }
$$

Consequently, $f=[f, \bar{f}] \in \mathbb{A}(X)$ is S-continuous if and only if $f$ is lower semi-continuous and $\bar{f}$ is upper semicontinuous. Therefore if we identify a continuous function $f \in \mathscr{C}(X)$ with the (degenerate) interval function $[f, f]$, it is clear that the resulting interval function is Scontinuous and, since its values are degenerate intervals, it is H-continuous. Conversely, if $f=[f, \bar{f}] \in \mathbb{H}(X)$ is such that $f(x)$ is a degenerate interval for every $x \in X$, then $f=\bar{f}$ is a continuous function. In this way we may identify $\mathscr{C}(X)$ in a canonical way with a subset of $\mathbb{H}(X)$, namely, the set of $\mathrm{H}$-continuous functions with degenerate values at every $x \in X$. We make no distinction between a continuous function $f$ and the interval function $[f, f]$ so that we consider $\mathscr{C}(X)$ as a subset of $\mathbb{H}(X)$.

Recall the following results on $\mathrm{H}$-continuous functions [1] which will be used in subsequent sections.

Theorem 2.3: The following are equivalent for all $f=$ $[f, \bar{f}] \in \mathbb{A}(X)$.

(i) $f$ is H-continuous.

(ii) $F(\underline{f})=F(\bar{f})=f$. 
(iii) $S(\underline{f})=\bar{f}$ and $I(\bar{f})=\underline{f}$.

Theorem 2.4: If $f=[\underline{f}, \bar{f}]$ is H-continuous, then the set

$$
D_{\varepsilon}(f)=\{x \in X: \bar{f}-\underline{f}<\varepsilon\}
$$

is open and dense in $X$ for every $\varepsilon>0$.

Theorem 2.5: The functions $F(S(I(f)))$ and $F(I(S(f))$ are H-continuous for every $f \in \mathbb{A}(X)$.

We may note that if $f=[f, \bar{f}]$ is S-continuous then, due to (4), we have

$$
F(S(I(f)))=F(S(\underline{f}))=[I(S(\underline{f})), S(\underline{f})] .
$$

Since

$$
\underline{f}=I(\underline{f}) \leq I(S(\underline{f})) \leq S(\underline{f}) \leq S(\bar{f})=\bar{f}
$$

it follows that

$$
F(S(I(f)))(x) \subseteq f(x), x \in X .
$$

Similarly, $F(I(S(f)))(x) \subseteq f(x), x \in X$.

\section{B. Interval Arithmetic}

We now recall the arithmetic operations on $\mathbb{R}$, see for instance [3]. The sum of two intervals $a=[\underline{a}, \bar{a}], b=$ $[\underline{b}, \bar{b}] \in \mathbb{R}$ is given by

$$
a+b=[\underline{a}+\underline{b}, \bar{a}+\bar{b}] .
$$

The scalar multiple of $a \in \mathbb{R}$ and $\alpha \in \mathbb{R}$ is defined as

$$
\alpha a=[\min \{\alpha \underline{a}, \alpha \bar{a}\}, \max \{\alpha \underline{a}, \alpha \bar{a}\}] .
$$

With respect to addition and scalar multiplication, defined in this way, $\mathbb{R}$ is not a vector space over $\mathbb{R}$. Indeed, the additive identity in $\mathbb{I} \mathbb{R}$ is clearly the degenerate interval $0=[0,0]$. However, if $a=[\underline{a}, \bar{a}] \in \mathbb{R}$ is a proper interval, that is $\underline{a} \neq \bar{a}$, then $a$ does not have an additive inverse. However, as is shown in [20], $\mathbb{I R}$ does have a rich algebraic structure. In fact, $\mathbb{I} \mathbb{R}$ is a quasilinear space with cancelation law. That is, the following properties are satisfied for all $a, b, c \in \mathbb{R}$ and $\alpha, \beta \in \mathbb{R}$.

(i) $a+(b+c)=(a+b)+c$

(ii) $a+b=b+a$

(iii) $a+b=a+c \Rightarrow b=c$

(iv) $\alpha(a+b)=\alpha a+\alpha b$

(v) $\alpha(\beta a)=(\alpha \beta) a$

(vi) $1 a=a$

(vii) $(\alpha+\beta) a=\alpha a+\beta a$ if $\alpha \beta \geq 0$

\section{The Linear Space $\mathbb{H}(X)$}

The linear structure of $\mathscr{C}(X)$ is defined, naturally, through the pointwise operations

$$
(f+g)(x)=f(x)+g(x), x \in X
$$

and

$$
(a f)(x)=a f(x), x \in X .
$$

The fact that these operations turn $\mathscr{C}(X)$ into a linear space over $\mathbb{R}$ is due to the fact that addition and multiplication are continuous operations form $\mathbb{R} \times \mathbb{R}$ to $\mathbb{R}$, and that $\mathbb{R}$ is a linear space over itself. The first condition implies that $\mathscr{C}(X)$ is closed under the operations of pointwise addition and scalar multiplication, while the second ensures that the resulting operations satisfy the axioms of a linear space.

For $f, g \in \mathbb{A}(X)$, let us denote by $f \oplus g$ the pointwise interval sum of $f$ and $g$. That is,

$$
\begin{aligned}
f \oplus g(x) & =f(x)+g(x) \\
& =[\underline{f}(x)+\underline{g}(x), \bar{f}(x)+\bar{g}(x)], x \in X .
\end{aligned}
$$

For $\alpha \in \mathbb{R}$, the pointwise product $\alpha \odot f$ is defined as

$$
\begin{aligned}
& \alpha \odot f(x) \\
& =\alpha f(x) \\
& =[\min \{\alpha \underline{f}(x), \alpha \bar{f}(x)\}, \max \{\alpha \underline{f}(x), \alpha \bar{f}(x)\}] .
\end{aligned}
$$

We note the following consequence of the fact that $\mathbb{I R}$ is a quasi-linear space over $\mathbb{R}$.

Proposition 3.1: With respect to addition and scalar multiplication given by $(7)$ and $(8), \mathbb{A}(X)$ is a quasilinear space with cancellation law.

When attempting to extend the pointwise operations on $\mathscr{C}(X)$ to $\mathbb{H}(X)$ in this way, one encounters a double breakdown of the above situation. Firstly, $\mathbb{H}(X)$ is not closed under the pointwise operation of interval addition.

Example 3.2: Consider the functions $f, g \in \mathbb{H}(\mathbb{R})$ given by

$$
f(x)=\left\{\begin{array}{lll}
0 & \text { if } & x<0 \\
{[0,1]} & \text { if } & x=0 \\
1 & \text { if } & x>0
\end{array}\right.
$$

and

$$
g(x)=\left\{\begin{array}{lll}
0 & \text { if } & x<0 \\
{[-1,0]} & \text { if } & x=0 \\
-1 & \text { if } & x>0
\end{array}\right.
$$


The pointwise sum of $f$ and $g$ is

$$
f \oplus g(x)=\left\{\begin{array}{lll}
0 & \text { if } & x \neq 0 \\
{[-1,1]} & \text { if } & x=0
\end{array}\right.
$$

which is clearly not $\mathrm{H}$-continuous.

It is easily seen that $\mathbb{F}(X)$ is closed under the pointwise interval operations. However, here we encounter the second breakdown: The pointwise interval operations on $\mathbb{F}(X)$ do not satisfy the axioms of a linear space. This is due to the fact that, as mentioned in Section II (B), $\mathbb{R}$ is not a vector space over $\mathbb{R}$.

The main result of this section, namely, that $\mathbb{H}(X)$ is a vector space over $\mathbb{R}$ for any topological space $X$, is a consequence of the following.

Proposition 3.3: If $f=[f, \bar{f}]$ is S-continuous and the set

$$
D_{\varepsilon}(f)=\{x \in X: \bar{f}(x)-\underline{f}(x)<\varepsilon\}
$$

contains an open and dense subset of $X$ for every $\varepsilon>0$, then $f$ contains exactly one $\mathrm{H}$-continuous function.

Proof: Since $f$ is S-continuous, it follows from (2), (3) and (5) that $f$ contains the functions $h_{1}=F(I(S(f)))$ and $h_{2}=F(S(I(\bar{f})))$, and $h_{1} \leq h_{2}$. According to Theorem 2.5 both $h_{1}$ and $h_{2}$ are H-continuous. Suppose that $g=$ $[g, \bar{g}]$ is H-continuous and $g(x) \subseteq f(x), x \in X$. Then $f \leq$ $\underline{g} \leq \bar{g} \leq \bar{f}$ so that the monotonicity of the operators $I$ and $S$ (2) and Theorem 2.3 implies that $h_{1} \leq g \leq h_{2}$. Therefore it is sufficient to shown that $h_{1}=h_{2}$. Suppose that there exists $x_{0} \in X$ so that $\underline{h}_{2}\left(x_{0}\right)>\bar{h}_{1}\left(x_{0}\right)$. Let $M=$ $\left(\underline{h}_{2}\left(x_{0}\right)+\bar{h}_{1}\left(x_{0}\right)\right) / 2$ so that $\underline{h}_{2}\left(x_{0}\right)>M>\bar{h}_{1}\left(x_{0}\right)$. Pick any $\varepsilon>0$ so that $\underline{h}_{2}\left(x_{0}\right)>M+\varepsilon / 2>M-\varepsilon / 2>\bar{h}_{1}\left(x_{0}\right)$. Since $\underline{h}_{2}$ is lower semi-continuous and $\bar{h}_{1}$ is upper semicontinuous, there exists $V \in \mathscr{V}_{x_{0}}$ so that $\underline{h}_{2}(x)>M+$ $\varepsilon / 2>M-\varepsilon / 2>\bar{h}_{1}(x)$ for every $x \in V$. Then $h_{2}(x)-$ $\bar{h}_{1}(x)>\varepsilon$ for every $x \in V$. According to 2 and $\overline{5}$,, $\bar{f}=$ $S(\bar{f}) \geq S(I(\bar{f}))=\bar{h}_{2} \geq \underline{h}_{2}$ and $f=I(f) \leq I(S(f))=\underline{h}_{1} \leq$ $\bar{h}_{1}$. Therefore $\bar{f}(x)-f(x) \geq \bar{h}_{2}(x)-\underline{h}_{1}(x)>\bar{\varepsilon}$ for every $x \in V$. Since $V$ is open, it follows that $D_{\varepsilon}(f)$ not dense in $X$, contrary to the assumption that $D_{\varepsilon}(f)$ contains an open and dense subset $X$. Therefore $\underline{h}_{2}(x) \leq \bar{h}_{1}(x)$ for every $x \in X$. Then, since $\bar{h}_{1}$ is upper semi-continuous and $\underline{h}_{2}$ is lower semi-continuous, (2) and Theorem 2.3 imply that

$$
\bar{h}_{2}=S\left(\underline{h}_{2}\right) \leq S\left(\bar{h}_{1}\right)=\bar{h}_{1}
$$

and

$$
\underline{h}_{2}=I\left(\underline{h}_{2}\right) \leq I\left(\bar{h}_{1}\right)=\underline{h}_{1}
$$

so that $h_{2} \leq h_{1}$. Hence $h_{1}=h_{2}$.
Corollary 3.4: If $f_{1}, \ldots, f_{n}$ are H-continuous, then the function $f_{1} \oplus \ldots \oplus f_{n}$ contains exactly one $\mathrm{H}$-continuous function.

Proof: We give a proof for the case when $n=2$. The general case follows in exactly the same way. Let $f=[f, \bar{f}]$ and $g=[g, \bar{g}]$ be H-continuous. Therefore $f$ and $\bar{g}$ are S-continuous, so that $f, g$ are lower semicontinuous while $\bar{f}, \bar{g}$ are upper semi-continuous. Since the sum of lower (upper) semi-continuous functions are lower (upper) semi-continuous, it follows that $f \oplus g=$ $[f \oplus g, \overline{f \oplus g}]=[f+g, \bar{f}+\bar{g}]$ is S-continuous. Fix $\varepsilon>0$. It follows from Theorem 2.4 that $D_{\varepsilon}(f \oplus g)=\{x \in$ $X: \overline{f \oplus g}(x)-f \oplus g(x)<\varepsilon\}$ contains an open and dense subset of $X$. In particular, $D_{\frac{\varepsilon}{2}}(f) \cap D_{\frac{\varepsilon}{2}}(g) \subseteq D_{\varepsilon}(f \oplus g)$. The result follows from Proposition 3.3 .

In view of Corollary 3.4 we define addition in $\mathbb{H}(X)$ as follows.

Definition 3.5: For $f, g \in \mathbb{H}(X)$, the sum $f+g$ of $f$ and $g$ is the unique $\mathrm{H}$-continuous function contained in $f \oplus g$.

Theorem 3.6: $\mathbb{H}(X)$ is a linear space over $\mathbb{R}$ with addition defined as in Definition 3.5 and scalar multiplication given by $(8)$. Furthermore, $\mathscr{C}(X)$ is a linear subspace of $\mathbb{H}(X)$.

Proof: Consider $f, g, h \in \mathbb{H}(X)$ and $\alpha, \beta \in \mathbb{R}$. Denote by 0 both the additive identity in $\mathbb{R}$ and the $\mathrm{H}$ continuous function that is identically 0 .

$\mathbb{H}(X)$ is closed under scalar multiplication. If $\alpha=0$, then $\alpha f=\alpha \odot f=0$, so $\alpha f$ is H-continuous. Suppose that $\alpha>0$. Clearly $\alpha f=[\alpha f, \alpha \bar{f}]$ is S-continuous. Suppose that $f_{1}=\left[f_{1}, \bar{f}_{1}\right] \subseteq \alpha \bar{f}$ is S-continuous. Then $\frac{1}{\alpha} f_{1}=\left[\frac{1}{\alpha} f_{1}, \frac{1}{\alpha} \bar{f}_{1}\right]$ is S-continuous and $\frac{1}{\alpha} f_{1} \subseteq f$ so that $\frac{1}{\alpha} f_{1}=f$. Hence $f_{1}=\alpha\left(\frac{1}{\alpha}\right) f_{1}=\alpha f$ so that $\alpha f$ is $\mathrm{H}$ continuous. The case when $\alpha<0$ is dealt with in the same way.

Associativity of addition. Since addition in $\mathbb{I} \mathbb{R}$ is associative, it follows that $f \oplus(g \oplus h)=(f \oplus g) \oplus h=$ $f \oplus g \oplus g$. Now $f+(g+h) \subseteq f \oplus(g+h) \subseteq f \oplus(g \oplus h)=$ $f \oplus g \oplus h$ and, similarly, $(f+g)+h \subseteq f \oplus g \oplus h$. It now follows from Corollary 3.4 that $f+(g+h)=(f+g)+h$. Commutativity of addition. Since $f+g \subseteq f \oplus g=$ $g \oplus f \supseteq g+f$, it follows from Corollary 3.4 that $f+g=$ $g+f$.

Additive identity. Clearly $f+0=f \oplus 0=f$.

Additive inverse. We have $f+(-1 f) \subseteq f \oplus(-1 f)=$ $[f-\bar{f}, \bar{f}-f]$ so that $0 \subseteq f \oplus(-1 f)$. Corollary 3.4 implies that $f+(-1 f)=0$.

First distributive law. According to Proposition 3.1. $\alpha(f+g) \subseteq \alpha(f \oplus g)=(\alpha f) \oplus(\alpha g) \supseteq \alpha f+\alpha g$. Thus 
$\alpha(f+g)=\alpha f+\alpha g$ by Corollary 3.4

Second distributive law. According to Definition 3.5 . $\alpha f+\beta f \subseteq \alpha f \oplus \beta f$. But by Proposition 3.1. $\alpha f \oplus \beta f=$ $(\alpha+\beta) f$ if $\alpha \beta \geq 0$ so that, in this case, $(\alpha+\beta) f=$ $\alpha f+\beta f$ by Definition 2.2. Now suppose that $\alpha>0$ and $\beta<0$. If $\alpha+\beta>0$, then

$\alpha \underline{f}+\beta \bar{f} \leq \alpha \underline{f}+\beta \underline{f}=(\alpha+\beta) \underline{f} \leq(\alpha+\beta) \bar{f} \leq \alpha \bar{f}+\beta \underline{f}$ so that $(\alpha+\beta) f \subseteq \alpha f \oplus \beta f$. Corollary 3.4 now implies that $(\alpha+\beta) f=\alpha f+\beta f$. The case when $\alpha+\beta \leq 0$ follows in the same way.

Associativity of scalar multiplication. It follows from Proposition 3.1 that $\alpha(\beta f)=(\alpha \beta) f$.

Multiplicative identity. It follows from (8) that $1 f=f$. If $f, g \in \mathscr{C}(X)$, then $f \oplus g=f+g$ so that $\mathscr{C}(X)$ is closed under addition in $\mathbb{H}(X)$, with the sum in $\mathbb{H}(X)$ corresponding to the pointwise sum in $\mathscr{C}(X)$. Furthermore, in view of $(8), \mathscr{C}(X)$ is closed under scalar multiplication. Therefore $\mathscr{C}(X)$, with the usual pointwise operations, is a linear subspace of $\mathbb{H}(X)$.

Finally we show that $\mathbb{H}(X)$ is an Archimedean vector lattice with respect to the partial order $(1)$, and that $\mathscr{C}(X)$ is a sublattice of $\mathbb{H}(X)$.

Theorem 3.7: $\mathbb{H}(X)$ is an Archimedean vector lattice with respect to the partial order 11$)$. Furthermore, $\mathscr{C}(X)$ with the usual pointwise order is a sublattice of $\mathbb{H}(X)$.

Proof: First we prove that $\mathbb{H}(X)$ is a lattice. Consider $f=[f, \bar{f}], g=[g, \bar{g}] \in \mathbb{H}(X)$ and let $h^{\prime}(x)=$ $\sup \{f(x), g(x)\}$ for every $\bar{x} \in X$. Since $h^{\prime}$ is lower semicontinuous, it follows from (2) and Theorems 2.3 and 2.5 that $h=F\left(S\left(h^{\prime}\right)\right) \in \mathbb{H}(X)$ and $f, g \leq h$. Suppose that $f, g \leq p$ for some $p \in \mathbb{H}(X)$. Then $f, g \leq p$ so that $h^{\prime} \leq p$. Theorem 2.3 and 2 imply that $\underline{h}=I\left(S\left(h^{\prime}\right)\right) \leq$ $I(S(\underline{p}))=p$. Similarly $\bar{h} \leq \bar{p}$ so that $h \leq p$. It therefore follows that $h=\sup \{f, g\}$ in $\mathbb{H}(X)$. In the same way $\inf \{f, g\}=F\left(I\left(h^{\prime \prime}\right)\right.$ where $h^{\prime \prime}(x)=\inf \{\bar{f}(x), \bar{g}(x)\}$ for every $x \in X$.

Next we show that $\mathbb{H}(X)$ is an Archimedean vector lattice. In this regard, consider $f, g, h \in \mathbb{H}(X)$ so that $f \leq g$ and a real number $\alpha>0$. Since $\alpha f=[\alpha \underline{f}, \alpha \bar{f}]$ and $\alpha g=[\alpha g, \alpha \bar{g}]$ it is clear that $\alpha f \leq \alpha g$. Since $f \oplus h=$ $[f+\underline{h}, \bar{f}+\bar{h}]$ is S-continuous, it follows from $\sqrt{6}$ that $\bar{F}(S(I(f+\underline{h})))(x) \subseteq f \oplus h(x), x \in X$. Since, by Corollary 3.4. $f \oplus h$ contains exactly one H-continuous function, namely $f+h$, and $F(S(I(f+\underline{h})))$ is H-continuous by Theorem 2.5, it follows that $f+h=F(S(I(f+\underline{h})))$. In the same way, $g+h=F(S(I(g+\underline{h})))$. Since $\underline{f}+\underline{h} \leq g+$ $\underline{h}$, it follows from (2) that $f+h \leq g+h$. Therefore $\mathbb{H}(X)$ is a vector lattice. Since $\mathbb{H}(X)$ is Dedekind complete [1], it follows from [18, Theorem 25.1] that $\mathbb{H}(X)$ is
Archimedean.

Lastly we show that $\mathscr{C}(X)$ is a vector lattice subspace of $\mathbb{H}(X)$. Since $\mathscr{C}(X)$ is a linear subspace of $\mathbb{H}(X)$, it is sufficient to show that $\mathscr{C}(X)$ is a sublattice of $\mathbb{H}(X)$, that is, we must show that the lattice operations in $\mathscr{C}(X)$ agree with those in $\mathbb{H}(X)$. This is clear from the respective expressions for $\sup \{f, g\}$ and $\inf \{f, g\}$ in $\mathbb{H}(X)$.

We may note that Theorems 3.6 and 3.7 are known, indirectly, for the class of so-called completely regular weak cb-spaces [19]. The Dedkind completion of an Archimedean Riesz space is an Archimedean Riesz space [18]. Anguelov [1] showed that if $X$ is a completely regular space, then the Dedekind completion of (the Archimedean Riesz space) $\mathscr{C}(X)$ is the set $\mathbb{H}_{c m}(X)$ of H-continuous functions majorised by a continuous function. Dăneţ [14] showed that $\mathbb{H}_{c m}(X)=\mathbb{H}(X)$ if and only if $X$ is a weak cb-space. Therefore, if $X$ is a completely regular weak cb-space, then $\mathbb{H}(X)$ is the Dedekind completion of the Archimedean Riesz space $\mathscr{C}(X)$, and is therefore an Archimedean Riesz space, and hence also a vector space over $\mathbb{R}$. Here we have given direct proofs of these algebraic results, in the most general case.

\section{CONCLUSION}

It has been shown that $\mathbb{H}(X)$ is a linear space, and in fact an Archimedean Riesz space, over $\mathbb{R}$ for an arbitrary topological space, generalising a result of Anguelov, Markov and Sendov [4]. However, this generalisation required a new approach since the method [4] relies on the fact that, for $X$ a Baire space, a function $f \in \mathbb{H}(X)$ is pointvalued and continuous on a dense subset of $X$. In the general case considered here, this property does not hold. Our method may also apply to more general situations, namely, to spaces of set-valued maps with values in a metrizable topological vector space.

The result presented in this paper also has implications for real analysis and the life sciences. In [2] it is shown that the rational completion of $\mathscr{C}(X)$ may be constructed as a space of (nearly finite) $\mathrm{H}$-continuous functions when $X$ is completely regular. Our method may be applied to generalise this result to arbitrary topological spaces. Furthermore, computations involving uncertainty, which occurs frequently when modeling phenomena in the life sciences, need not be restricted to functions with domain a Baire space. 


\section{REFERENCES}

[1] R. Anguelov, Dedekind order completion of $C(X)$ by Hausdorff continuous functions. Quaestiones Mathematicae 27, 153 - 170 (2004). http://dx.doi.org/10.2989/16073600409486091

[2] R. Anguelov, The rational extension of $\mathscr{C}(X)$ via Hausdorff continuous functions. Thai Journal of Mathematics 5 no 2, 267 - 272 (2007).

[3] R. Anguelov and S. Markov, Extended segment analysis. Freiburger Interval Berichte 10, 1 - 63 (1981).

[4] R. Anguelov R, S. Markov, B. Sendov, The set of Hausdorff continuous functions - the largest linear space of interval functions. Reliable Computing 12, 337 - 363 (2006). http://dx.doi.org/10.1007/s11155-006-9006-5

[5] R. Anguelov and E. E. Rosinger, Solving large classes of nonlinear systems of PDEs. Computers and Mathematics with Applications 53, 491 - 507 (2007). http://dx.doi.org/10.1016/j.camwa.2006.02.040

[6] R. Anguelov and J. H. van der Walt, Order convergence structure on $\mathscr{C}(X)$. Quaestiones Mathematicae 28, 425 - 457 (2005). http://dx.doi.org/10.2989/16073600509486139

[7] R. Anguelov and J. H. van der Walt, Algebraic and topological structure of some spaces of set-valued maps. Computers and Mathematics with Applications 66, 1643-1654 (2013). http://dx.doi.org/10.1016/j.camwa.2013.04.008

[8] Z. Artstein, Piecewise Linear Approximations of Set-Valued Maps. Journal of Approximation Theory 56, 41 - 47 (1989). http://dx.doi.org/10.1016/0021-9045(89)90131-7

[9] Z. Artstein, Extensions of Lipschitz Selections and an Application to Differential Inclusions. Nonlinear Analysis: Theory, Methods and Applications 16, 701 - 704 (1991) http://dx.doi.org/10.1016/0362-546X(91)90176-2

[10] Z. Artstein, A calculus for set-valued maps and set-valued evolution equations. Set-Valued Analysis 3, 213 - 261 (1995). http://dx.doi.org/10.1007/BF01025922

[11] R. Baire, Lecons sur les fonctions discontinues. Collection Borel, Paris, 1905.

[12] A. Cellina, On the differential inclusion $x^{\prime} \in(-1,1)$, Rend. Acc. Naz. Lincei 69, 1 - 6 (1980).

[13] F. H. Clarke, Optimization and nonsmooth analysis. John Wiley \& Sons, New York, 1983.
[14] N. Dăneț, When is every Hausdorff continuous interval-valued function bounded by continuous functions? Quaestiones Mathematicae 32, 363-369 (2009). http://dx.doi.org/10.2989/QM.2009.32.3.7.908

[15] D. de Pereda, S. Romero-Vivo, J. Bondia, On the Computation of Output Bounds for Compartmental in-Series Models under Parametric Uncertainty. Biomath 1 1210043, 85 - 90 (2012). http://dx.doi.org/10.11145/j.biomath.2012.10.043

[16] A. F. Filippov, Differential equations with discontinuous righthand side. Nauka, Moskow, 1985 (in Russian).

[17] F. Lempio, V. Veliov, Discrete Approximation of Differential Inclusions. Bayreuther Mathematische Schriften 54, 149 - 232 (1998).

[18] W. A. J. Luxemburg and A. C. Zaanen, Riesz spaces I. North Holland, Amsterdam, 1971.

[19] J. E. Mack, On a class of countably paracompact spaces. Proceedings of the American Mathematical Society 16, 467 472 (1965). http://dx.doi.org/10.1090/S0002-9939-1965-0177388-1

[20] S. Markov, On the algebraic properties of intervals and some applications. Reliable Computing 7, 113 - 127 (2001). http://dx.doi.org/10.1023/A:1011418014248

[21] S. Markov, Biomathematics and interval analysis: a prosperous marriage. In: M. D. Todorov, Ch. I. Christov, Eds., AIP Conference Proceedings, Vol. 1301, Amitans'2010, American Institute of Physics, Melville, New York, 2010, pp. 26-36. http://dx.doi.org/10.1063/1.3526621

[22] R. E. Moore, Interval Analysis. Prentice Hall, Englewood Cliff, 1966.

[23] R. E. Moore, Methods and Applications of Interval Analysis. SIAM, Philadelphia, 1979.

[24] E. Popova, Explicit Description of AE Solution Sets for Parametric Linear Systems. SIAM. J. Matrix Anal. \& Appl. 33 (4), 1172 - 1189 (2012). http://dx.doi.org/10.1137/120870359

[25] R. T. Rockafellar, Convex analysis. Princeton University Press, Princeton, 1970.

[26] B. Sendov, Hausdorff Approximations. Kluwer, Dordrecht, 1990. 